Tropical Journal of Pharmaceutical Research, October 2009; 8 (5): 433-440

(C) Pharmacotherapy Group,

Faculty of Pharmacy, University of Benin

Benin City, 300001 Nigeria.

All rights reserved.

Research Article

Available online at http://www.tjpr.org

\title{
Alginate-Chitosan Particulate System for Sustained Release of Nimodipine
}

\section{Adhiyaman Rajendran*1 and Sanat Kumar Basu ${ }^{2}$}

${ }^{1}$ School of Pharmacy and Health Sciences, International Medical University, Kuala Lumpur, Malaysia, ${ }^{2}$ Division of Pharmaceutics, Department of Pharmaceutical Technology, Jadavpur University, Kolkata, India

\begin{abstract}
Purpose: The aim of this work was to prepare nimodipine-loaded alginate-chitosan beads for sustained drug release.

Methods: Nimodipine-loaded alginate-chitosan beads were prepared by ionic gelation method using various combinations of chitosan and $\mathrm{Ca}^{2+}$ as cations and alginate as anion. The swelling ability and in vitro drug release characteristics of the beads were studied at $\mathrm{pH} 1.2$ and 6.8. Infra-red (IR) spectrometry, scanning electron microscopy (SEM), differential scanning calorimetry (DSC), x-ray diffraction (XRD), and atomic absorption spectroscopy (AAS) were also applied to investigate the physicochemical characteristics of the drug in bead formulations.

Results: The surface morphology, size, and drug loading of the beads varied with increase in the concentration of chitosan and calcium chloride in the gelation medium. The swelling ability of the beads in different $\mathrm{pH}$ media was dependent on the presence of a polyelectrolyte complex in the beads and the $\mathrm{pH}$ of the media. Both calcium alginate beads and the beads treated with chitosan failed to release the drug at $\mathrm{pH} 1.2$ over the period of study. On the other hand, at $\mathrm{pH} 6.8$, calcium alginate beads released approx. $96 \%$ of drug in $6 \mathrm{~h}$, but treatment of the beads with chitosan lowered drug release to $73 \%$. Drug release mechanism was either "anomalous transport" or "case-Il transport". Data from characterisation studies indicate that there was no significant change in the physical state of the drug in the bead formulations.
\end{abstract}

Conclusion: Although nimodipine-loaded alginate beads showed poor sustained release characteristics, modification with chitosan yielded beads that exhibited sustained drug release.

Key words: Alginate-chitosan beads; Nimodipine; Swelling; Physicochemical characterization; Sustained release. 


\section{INTRODUCTION}

Alginate is a natural biopolymer which forms a hydrogel in the presence of divalent cations such as $\mathrm{Ca}^{2+}[1]$. The inert environment within the biopolymer network of alginates allows for the entrapment of a wide range of substances [2]. Much attention has been given in recent years to the use of chitosanalginate polyelectrolyte complex in controlled drug delivery [3]. The use of chitosan has been reported in the literature for coating alginate beads in order to alter the diffusion rate of the encapsulated substances and also as an additive for the bulk modification of the bead structure [4].

Nimodipine (NM) is isopropyl-2-methoxyethyl1,4-dihydro-2,6-dimethyl-4-(3-nitrophenyl)-3,5 -pyridine dicarboxylate, a dihydropyridine calcium antagonist. It has a short half-life of $1-2 \mathrm{~h}$, and the usual oral dose is 30 to $60 \mathrm{mg}$ to be taken 2 to 4 times a day. Thus, nimodipine is a suitable candidate for oral sustained release drug delivery. In this work, the effect of calcium ion and chitosan in the coagulation fluid on drug release characteristics as well as the release mechanism were evaluated. Also, the physical state of the drug in the beads and its swelling behavior in the bead formulation were assessed.

\section{EXPERIMENTAL}

\section{Materials}

The following materials were used as received: sodium alginate (low viscosity; viscosity of $2 \%$ solution $25{ }^{\circ} \mathrm{C}, \approx 250 \mathrm{cps}$, from SNAP Natural and Alginate Products Limited, Ranipet, India); chitosan (85\% degree of deacetylation, molecular weight > $10^{3} \mathrm{kD}$, from India Sea Foods, Cochin, India); calcium chloride dihydrate (Qualigens, Mumbai, India); and di-sodium hydrogen phosphate anhydrous, potassium dihydrogen phosphate, sodium acetate, potassium chloride, sodium hydroxide, dichloromethane, ethanol (99\%), hydrochloric acid and glacial acetic acid (all from Merck, Mumbai, India). Nimodipine B.P. was obtained as a gift from Cipla Limited, Mumbai, India.

\section{Preparation of nimodipine-loaded calcium alginate beads}

Nimodipine was dissolved in dichloromethane and then slowly dispersed in sodium alginate solution with constant stirring for $3 \mathrm{~h}$, as per the composition outlined in Table 1 . The dispersion was added drop-wise to the gelation medium (either $2 \%$ or $5 \% \mathrm{w} / \mathrm{v}$ calcium chloride) using a $5 \mathrm{ml}$ hypodermic syringe fitted with a $21^{\circ}$-gauge needle and with the gelation medium stirred constantly at room temperature $\left(25^{\circ} \mathrm{C}\right)$. The beads were left to cure in the gelation medium for $4 \mathrm{~h}$, taken out, washed with distilled water twice and then dried at $30{ }^{\circ} \mathrm{C}$ in a dust free chamber until they attained constant weight. Two drug : sodium alginate ratios $-1: 3$ and $1: 4$ - were used in the preparation of the beads. The beads were labeled CAB.

In another batch labeled ACB-I, drug-loaded alginate-chitosan beads were prepared using chitosan solution $(0.5 \%$ or $0.75 \% \quad \mathrm{w} / \mathrm{v})$ containing calcium chloride ( $2 \%$ or $5 \% \mathrm{w} / \mathrm{v}$ ) as the gelation medium. The vehicle of the chitosan solution was $3 \% \mathrm{v} / \mathrm{v}$ acetic acid and the $\mathrm{pH}$ of the medium was adjusted to $4.5 \pm$ 0.1 with $0.1 \mathrm{M}$ sodium hydroxide. Drug: polymer ratio was varied as described above. The gelation medium was prepared $2 \mathrm{~h}$ before use. The beads were prepared and recovered as described earlier.

In another variation, ACB-II beads were prepared as for ACB-I except that the cure time was $2 \mathrm{~h}$ instead of $4 \mathrm{~h}$. In a further variation, multilayered $(\mathrm{MB})$ beads prepared as for ACB-II (at a chitosan concentration of $0.75 \%$ ) were first treated with sodium alginate solution $(0.1 \% \mathrm{w} / \mathrm{v})$ and then cured in calcium chloride solution $(1 \% \mathrm{w} / \mathrm{v})$ for $30 \mathrm{~min}$ at room temperature $\left(25^{\circ} \mathrm{C}\right)$. 
Table 1: Composition of nimodipine-loaded calcium alginate (Nm-CAB), alginate-chitosan (Nm-ACB-I \& II) and multilayer (Nm-MB) beads

\begin{tabular}{|c|c|c|c|c|c|c|}
\hline \multirow{2}{*}{$\begin{array}{l}\text { Formulation Code } \\
\text { (Drug:polymer ratio) } \\
(1: 3 / 1: 4)\end{array}$} & \multicolumn{2}{|c|}{ Gelation medium } & \multicolumn{4}{|c|}{ Post-gelation treatment } \\
\hline & $\begin{array}{c}\mathrm{CaCl}_{2} \\
(\% \mathrm{w} / \mathrm{v})\end{array}$ & $\begin{array}{c}\mathrm{CH} \\
(\% \mathrm{w} / \mathrm{v})\end{array}$ & $\begin{array}{c}\mathrm{CH} \\
(\% \mathrm{w} / \mathrm{v})\end{array}$ & $\begin{array}{l}\mathrm{CaCl}_{2} \\
(\% \mathrm{w} / \mathrm{v})\end{array}$ & $\begin{array}{c}\text { SA } \\
(\% \mathrm{w} / \mathrm{v})\end{array}$ & $\begin{array}{c}\mathrm{CaCl}_{2} \\
(\% \mathrm{w} / \mathrm{v})\end{array}$ \\
\hline $\mathrm{A} 0 / \mathrm{AOa}$ & 2 & - & - & - & - & - \\
\hline A1/A1a & 2 & 0.5 & - & - & - & - \\
\hline $\mathrm{A} 2 / \mathrm{A} 2 \mathrm{a}$ & 2 & 0.75 & - & - & - & - \\
\hline АЗ/АЗа & 2 & - & 0.5 & 1 & - & - \\
\hline $\mathrm{A} 4 / \mathrm{A} 4 \mathrm{a}$ & 2 & - & 0.75 & 1 & - & - \\
\hline A5/A5a & 2 & - & 0.75 & 1 & 0.1 & 1 \\
\hline $\mathrm{FA0} / \mathrm{FAOa}$ & 5 & - & - & - & - & - \\
\hline FA1/FA1a & 5 & 0.5 & - & - & - & - \\
\hline $\mathrm{FA} 2 / \mathrm{FA} 2 \mathrm{a}$ & 5 & 0.75 & - & - & - & - \\
\hline FA3/FAЗа & 5 & - & 0.5 & 1 & - & - \\
\hline FA4/FA4a & 5 & - & 0.75 & 1 & - & - \\
\hline FA5/FA5a & 5 & - & 0.75 & 1 & 0.1 & 1 \\
\hline
\end{tabular}

Note: Sodium alginate was $5 \% \mathrm{w} / \mathrm{v}$ in all formulations; $\mathrm{CH}=$ chitosan; $\mathbf{C a C l}_{2}=$ calcium chloride; $S A=$ sodium alginate

\section{Morphological characterisation and size of beads}

The beads were examined by a scanning electron microscope (JEOL JSM - 5200, Japan) operating between $5-24 \mathrm{kV}$. The specimens were mounted on a metal stub (with double-sided adhesive tape) and coated under vacuum with gold in nitrogen atmosphere prior to observation. The bead size of all the formulations was determined by optical microscopy. At least 100 beads were analyzed for each preparation and the mean particle size was calculated.

\section{Determination of drug loading}

An accurately weighed sample $(10 \mathrm{mg})$ of drug-loaded bead was pulverized using mortar and pestle, incubated in $10 \mathrm{ml} 0.02 \mathrm{M}$ phosphate buffer $(\mathrm{pH}$ 6.8) at room temperature for $24 \mathrm{~h}$ for complete digestion and the drug extracted with ethanol (99\% $\mathrm{v} / \mathrm{v})$. The solution was filtered through a filter disc (particle retention: $11 \mu \mathrm{m}$ ) and then the filtrate was assayed spectrophotometrically for drug content at $236 \mathrm{~nm}$. The same method was utilized to prepare the blank but using unloaded beads. All the experiments were performed in triplicate. Drug loading or incorporation efficiency (\%) was calculated using the formula in Eq 1.

Experimental drug loading in \% $(E L)=L / L_{0} X$ 100

where $L$ is the actual drug content of the beads and $L_{0}$ is the weight of the beads.

\section{Swelling studies}

The swelling properties of the calcium alginate, alginate-chitosan and alginatechitosan multilayer beads were determined in buffer solutions of $\mathrm{pH} \mathrm{1.2,4.5,} \mathrm{and} \mathrm{6.8,}$ respectively, at predetermined time intervals. A sample of beads of known weight $(10 \mathrm{mg})$ was placed in a Petri dish containing $20 \mathrm{ml}$ of the buffer solution and allowed to swell at room temperature. At hourly intervals, the beads were removed and weighed. The weight of the swollen beads was determined (after blotting with filter paper to remove surface fluid) on an electronic balance (AB204-S Mettler Toledo), and thereafter 
returned to the buffer solution. The swelling ratio of the beads was calculated from the formula given in Eq 2 [5].

$$
\text { Swelling ratio }=W_{t} / W_{0}
$$

where $W_{t}$ is the weight of the swollen beads and $W_{0}$ is the initial weight of the beads.

\section{Determination of calcium content}

The calcium content of blank calcium alginate and alginate-chitosan beads, as well as that of nimodipine-loaded beads was determined using the procedure of Takka $S$ et $\mathrm{al}^{7}$. Beads $(100 \mathrm{mg})$, accurately weighed, were dissolved in $2 \mathrm{ml}$ of concentrated nitric acid by boiling. The sample was made up to $10 \mathrm{ml}$ with $1 \% \mathrm{v} / \mathrm{v}$ nitric acid and the calcium content was determined by atomic absorption spectroscopy (Perkin Elmer, model Analysis200).

\section{In vitro drug release studies}

Each sample for release studies consisted of the beads, equivalent to $20 \mathrm{mg}$ of nimodipine, and filled into a hard gelatin capsule. Drug release studies were carried out, using a USP dissolution rate test apparatus type II (Electro Lab model TDT- 08L), in $600 \mathrm{ml}$ of hydrochloric acid buffer solution $(\mathrm{pH} \mathrm{1.2)} \mathrm{or}$ phosphate buffer solution ( $\mathrm{pH}$ 6.8) for $4 \mathrm{~h}$ and $6 \mathrm{~h}$, respectively, at $37 \pm 0.5^{\circ} \mathrm{C}$. The dissolution media also contained $0.3 \% \mathrm{w} / \mathrm{v}$ of sodium dodecyl sulphate (SDS) to maintain sink conditions for the drug. The apparatus was operated at a stirring speed of $100 \mathrm{rpm}$. Five (5) $\mathrm{ml}$ of the dissolution medium was sampled at predetermined time intervals, and replenished with the same quantity of fresh dissolution medium on each occasion to keep the volume constant. The sample was filtered through a filter disc (particle retention: $11 \mu \mathrm{m}$ ) and analyzed for drug content at $236 \mathrm{~nm}$ on a spectrophotometer (160-UV-visible Shimadzu spectrophotometer). The release kinetics of nimodipine from the beads were also evaluated using different models, zero order, first order, Higuchi, Peppas-Korsmeyer and Hixon-Crowell. This assessment was carried out with the aid of a program, PCP Disso v
2.08 (Anant Katkar, Poona College of Pharmacy, India).

\section{FT-Infrared spectroscopy}

Individual beads/samples were crushed in a mortar with a pestle. The crushed material was mixed with potassium bromide (Merck IR spectroscopy grade) in a 1:100 proportion and dried at $40{ }^{\circ} \mathrm{C}$. The mixture was compressed to a $12 \mathrm{~mm}$ semitransparent disk by applying a pressure of 10 tons for 2 minutes. The FTIR spectra over the wavelength range 4000 to $400 \mathrm{~cm}-1$ were recorded using a FTIR spectrometer (Perkin Elmer 1600 series).

\section{Differential scanning calorimetry}

Differential scanning calorimetry (DSC) nimodipine, sodium alginate, chitosan, as well as nimodipine/sodium alginate physical mixture (PM1), nimodipine/sodium alginate/chitosan physical mixture (PM2), nimodipine-loaded calcium alginate beads (A0) and alginate-chitosan beads-II (A4). The test was carried out using a thermal analysis system (Mettler TA 4000), calibrated with indium as the standard and operated in the temperature range, $30-400{ }^{\circ} \mathrm{C}$. The bead sample $(5 \mathrm{mg})$ which was heated at $10^{\circ} \mathrm{C} / \mathrm{min}$ in an aluminum pan under a nitrogen atmosphere using an empty pan as the reference. The automatically computed onsets of melting point and enthalpy of fusion were noted.

\section{X-ray diffraction}

$X$-diffraction patterns of the individual materials, physical mixtures and beads were obtained with an x-ray diffractometer (Rich Seifert, model $3000 \mathrm{P}$ ) at $30 \mathrm{kV}$ and $15 \mathrm{~mA}$ over a range of 10-100 20, using $\mathrm{Cu} \mathrm{Ka}$ radiation wavelength of $1.5405 \mathrm{~A}^{\circ}$. In the technique, the cavity of the metal sample holder of the equipment was filled with ground sample powder and then smoothed out with a spatula before the test was run.

Trop J Pharm Res, October 2009; 8 (5):436 


\section{RESULTS}

\section{Morphological characteristics and bead size}

The scanning electron micrograph of a typical drug-loaded alginate bead treated with chitosan is shown in Fig 1. The beads had a spherical shape and showed surface cracks probably caused by partial collapsing of the polymer network during drying. The mean particle size of the beads ranged from $1486.00 \pm 0.02$ to $1602.00 \pm 0.01 \mu \mathrm{m}$.

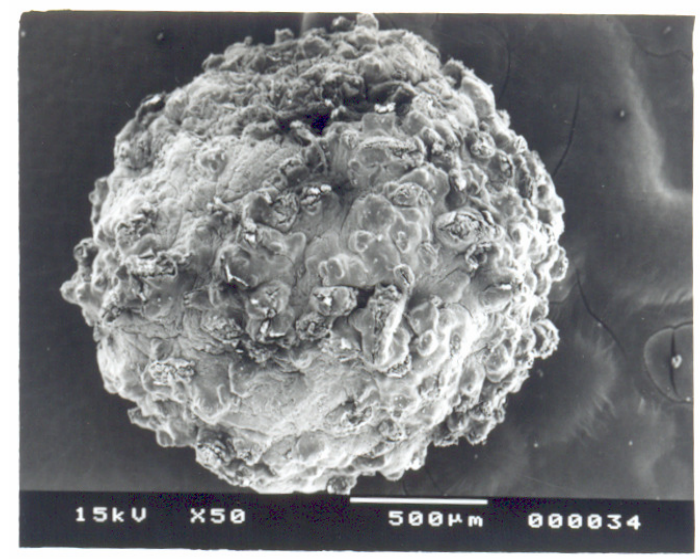

Figure 1: Scanning electron micrograph of nimodipine-loaded alginate/chitosan bead.

\section{Drug loading}

Drug loading was approx. 23 and $18 \%$ for formulations in which the drug/polymer ratio was $1: 3$ and $1: 4$, respectively. Variation in the treatment of the beads did not affect drug loading.

\section{Swelling characteristics of nimodipine- loaded beads}

The results of swelling studies, shown in Fig 2 , indicate that swelling varied with the $\mathrm{pH}$ of the medium. Maximum swelling ratios were as high as 2, 14 and 36 in media $\mathrm{pH}$ of 1.2 , 4.5 and 6.8 , respectively. The beads did not show any sign of disintegration in the media at $\mathrm{pH} 1.2$ and 4.5 over a period of $8 \mathrm{~h}$. However the swelling ratio of the beads at $\mathrm{pH}$ 4.5 was considerably greater than that at $\mathrm{pH}$ 1.2. Treatment of the calcium alginate beads with chitosan enhanced swelling at $\mathrm{pH}$ of 1.2 and 4.5. On the other hand, the beads began to slowly disintegrate in $\mathrm{pH} 6.8$ medium after $7 \mathrm{~h}$.

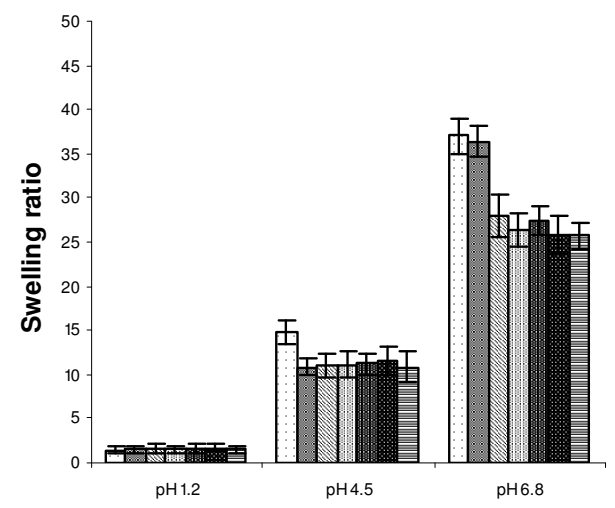

Swelling medium

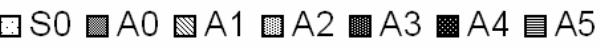

Figure 2: The swelling characteristics of nimodipine beads in various $\mathrm{pH}$ media. (Mean \pm $S D ; n=3$ ). Key: Blank calcium-alginate beads (SO), Drug loaded calcium-alginate beads (A0), Drug loaded alginate-chitosan beads prepared by the drug polymer dispersion directly dropped in to the gelation medium containing a mixture of calcium chloride with $0.5 \%$ chitosan solution (A1) and $0.75 \%$ chitosan solution (A2), Drug loaded alginate-chitosan beads prepared by incubate the drug loaded calcium-alginate beads in to the gelation medium containing a mixture of calcium chloride with $0.5 \%(\mathrm{w} / \mathrm{v})$ chitosan solution (A3) and $0.75 \%(\mathrm{w} / \mathrm{v})$ chitosan solution (A4), Alginatechitosan beads (A4) further incubated in $0.1 \%$ sodium alginate solution (A5).

\section{In vitro drug release of nimodipine}

At $\mathrm{pH} 1.2$, there was no discernible release of nimodipine from calcium alginate beads and those pretreated with chitosan after $4 \mathrm{~h}$. On the other hand, as Fig 3 illustrates, $95.8 \%$ of nimodipine was released from the untreated beads (formulation $\mathrm{A} 0$ ) at $\mathrm{pH} 6.8$ in $6 \mathrm{~h}$ during which it completely disintegrated. Following 
treatment with $0.75 \%$ chitosan, yielding formulation A4, drug release fell to $75.6 \%$. Further treatment with alginate, resulting in formulation A5, slightly reduced drug release further to $73.1 \pm 1.69 \%$.

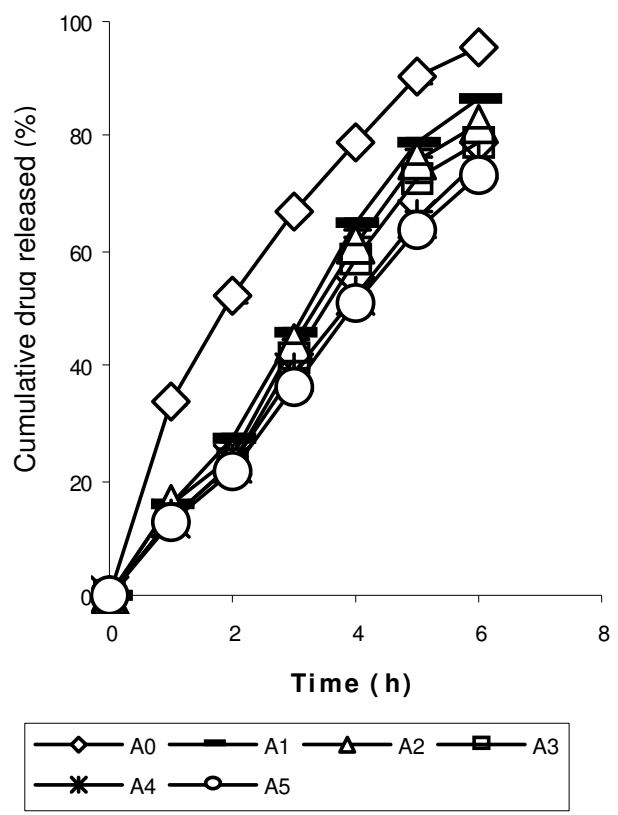

Figure 3: Cumulative release of nimodipine in $\mathrm{pH}$ 6.8 phosphate buffer from nimodipine-loaded beads (Mean \pm S.D.; $n=3$ ). Key: As for Fig 2

On subjecting the release data to various models, correlation coefficient $\left(r^{2}\right)$ ranged from $0.98-0.99$ for most of the formulations, except for the Higuchi model where was as low as 0.94 for some of the formulations. Furthermore, in the case of the Peppaskorsmeyer model, the release kinetics were also found to fit the classical power law expression, with $n$ values of $0.57-0.58$ for the untreated beads, and $0.87-0.93$ for the treated beads.

\section{FT-IR spectroscopy}

The FT-IR spectra (not displayed) of nimodipine alone manifested major peaks in the wave number range of 1000 to $1400 \mathrm{~cm}^{-1}$, indicating the presence of carboxyl and carboxylate groups, and $\mathrm{C}-\mathrm{H}$ stretching, appeared at 2981.48 to $2898.38 \mathrm{~cm}^{-1}$. Crosslinking of alginate by $\mathrm{Ca}^{2+}$ resulted in a decrease in the wave number of the carbonyl peak from 1623.7 to $1608.97 \mathrm{~cm}^{-1}$. The principal peaks of nimodipine were observed in the spectra of all the formulations.

\section{Differential scanning calorimetric analysis}

Fig 4 shows the DSC thermograms of nimodipine alone, the polymers, physical mixtures of the polymers and the drug, as well as nimodipine-loaded beads. A sharp endothermic peak corresponding to the melting of crystalline nimodipine was found at $126.8^{\circ} \mathrm{C}$ while sodium alginate decomposed at $253.6{ }^{\circ} \mathrm{C}$ with a broad exotherm. The degradation exotherm of sodium alginate was absent in formulations $A 0(C A B)$ and $A 4$ (ACB II) beads but an endotherm corresponding to the interaction of alginate with calcium ion was observed at $192.3^{\circ} \mathrm{C}$ and $187.7^{\circ} \mathrm{C}$, respectively. The melting endotherm of nimodipine in PM1 (physical mixture of nimodipine/sodium alginate), and PM2 (nimodipine/sodium alginate/chitosan) appeared at 126.0 and $127.6^{\circ} \mathrm{C}$, respectively.

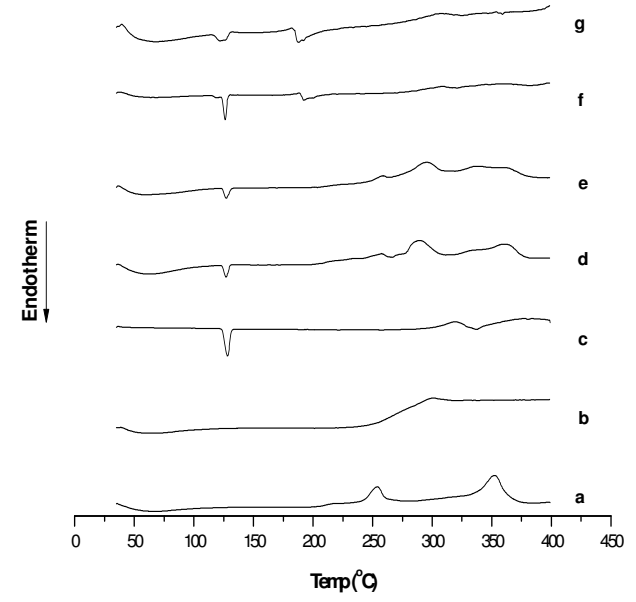

Figure 4: DSC thermograms of sodium alginate (a), chitosan (b), nimodipine (c), physical mixture of nimodipine with sodium alginate (d), physical mixture of nimodipine, sodium alginate and chitosan (e), nimodipine-loaded alginate beads (f), and nimodipine-loaded alginate beads coated with chitosan $(\mathrm{g})$ 
In the bead formulations, the melting endotherm of nimodipine appeared at 125.9 and $123.2{ }^{\circ} \mathrm{C}$ for formulations $\mathrm{A} 0$ and $\mathrm{A} 4$, respectively.

\section{X-ray diffraction (XRD)}

The x-ray diffractogram of nimodipine alone, the individual polymers, physical mixtures of the polymers and the drug, as well as the nimodipine-loaded beads are displayed in Fig 5. Pure nimodipine showed the classical diffractogram of the crystalline substance; that of sodium alginate indicated the presence of a completely amorphous material while the diffractogram of chitosan manifested a few crystalline peaks of very low intensity. The XRD pattern of the physical mixtures showed no changes in the crystalline nature of the individual components, but their peaks were more diffused in bead formulations, possibly due to changes in the degree of crystallinity of the drug that might have occurred following its dispersion in the polymer matrix of the beads ${ }^{8}$.

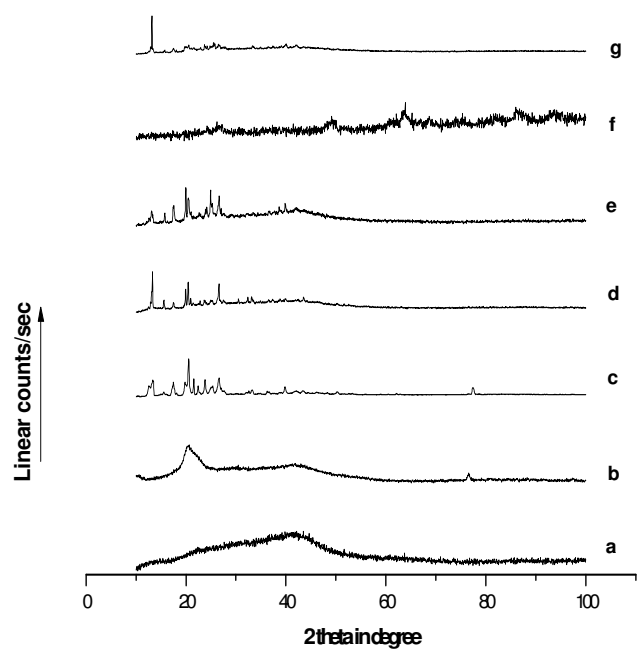

Figure 5: X-ray powder diffraction patterns of sodium alginate (a), chitosan (b), nimodipine (c), physical mixture of nimodipine with sodium alginate (d), physical mixture of nimodipine, sodium alginate and chitosan (e), nimodipineloaded alginate beads (f), and nimodipine-loaded alginate beads coated with chitosan $(\mathrm{g})$

\section{DISCUSSION}

Bead size was not noticeably enhanced following treatment of the alginate beads with chitosan. Further treatment with alginate also did not alter bead dimensions as would have been expected.

At low pH (1.2 and 4.5), bead swelling was minimal. This may be attributed to the inability of the medium to penetrate the beads at $\mathrm{pH} 1.2$, and limited penetration at $\mathrm{pH} 4.5$. On the other hand, the pronounced swelling of the beads in phosphate buffer $(\mathrm{pH}$ 6.8) followed by slow disintegration after $7 \mathrm{~h}$ might have been influenced by certain factors. It can be said that in the initial phase of the swelling process, the $\mathrm{Ca}^{2+}$ ions present in the polymannuronate units of alginate are exchanged with $\mathrm{Na}^{+}$ions present in the buffer solution, which ultimately causes chain relaxation and enhances gel formation or swelling. In the later stage of the swelling process, $\mathrm{Ca}^{2+}$ ions bind with the $-\mathrm{COO}^{-}$group of the polyglucuronate units and thus form the tight egg-box structure which also starts to exchange with $\mathrm{Na}^{+}$ions in the buffer medium because polyglucuronate sequences have a strong auto-cooperative binding of $\mathrm{Ca}^{2+}$ ions. Increase in the proportion of calcium alginate in the drug-loaded beads may led to a corresponding increase in the amount of $\mathrm{Ca}^{++}$, and hence availability of a larger number of binding sites for interaction with anions. It was observed that the amount of calcium ions in formulations (A4, A4a and FA4a) decreased when compared with formulation $\mathrm{A} 0$. This indicates that the alginate formed a complex both with calcium ions and chitosan [7].

Poor drug release at $\mathrm{pH} 1.2$ may be attributed to the poor swelling of the alginate beads at acidic $\mathrm{pH}$ due to the inability of the dissolution medium to penetrate the beads. However, the dissolution medium readily penetrated the beads at $\mathrm{pH} 6.8$, easily leaching out the drug incorporated in them. Treatment of the alginate beads with chitosan, and then with alginate, substantially 
reduced drug release. The probable reason is that electrostatic interaction between carboxyl groups of the alginate and the amino group of chitosan produced a compact surface layer that reduced both diffusion of fluid into the beads and the erosion of the beads. Drug release at $\mathrm{pH} 6.8$ was accompanied by rapid swelling and erosion/disintegration of the alginate beads. The drug release data for the various calcium alginate beads fitted into the classical power law expression, and the values of $n$ were around 0.6. This indicates that drug release from alginate beads followed non-Fickian kinetics, due probably to rapid swelling and erosion of the beads. The release data for alginate-chitosan (Nm-ACB-I \&II) multilayer beads also fitted well into the power law expression, and the values of $n$ decreased below 1 . It is likely that the formation of polyelectrolyte complex membrane reduced the initial swelling and erosion of the beads and shifted the drug release mechanism toward anomalous transport or case II transport, indicating that the drug was diffusing through the beads at the same time as polymer relaxation was taking place. Since the release of the drug from alginate-chitosan beads exhibited a small time lag, the release data were fitted into the modified power law expression, and this yielded excellent linearity. Consequently, it can be concluded that the drug release mechanism was anomalous diffusion type or case II transport.

FT-IR and DSC data indicate there was no interaction between nimodipine on the one hand, and chitosan and alginate on the other. For the DSC results, the slight differences observed in the melting endotherms and peak intensities may be attributed to homogeneous dispersion of the drug in the polymers and to variation in drug/polymer ratio. The differences observed between the XRD patterns of the bead formulations and those of the physical mixtures of the drug and polymers was probably due to a decrease in the degree of crystallinity of the drug, which resulted from its dispersion in the polymer matrix [8].

\section{CONCLUSION}

The study demonstrates that while alginate beads incorporating nimodipine exhibited comparatively rapid drug release at intestinal $\mathrm{pH}$ (6.8), treatment with chitosan alone or together with alginate lowered drug release. The bead formulations thus afford an option for achieving modulated release of the drug.

\section{REFERENCES}

1. Bajpai SK, Sharma S. Investigation of swelling/degradation behavior of alginate beads crosslinked with $\mathrm{Ca}^{2+}$ and $\mathrm{Ba}^{2+}$ ions. React. Funct. Polym 2004; 59: 129-140.

2. Rasmussen MR, Snabe T, Pedersen LH. Numerical modelling of insulin and amyloglucosidase release from swelling $\mathrm{Ca}$-alginate beads $\mathrm{J}$. Control. Release 2003; 91: 395-405.

3. Lee $K Y$, Park WH. Polyelectrolyte complexes of sodium alginate with chitosan or its derivatives for microcapsules. J. Appl. Polym. Sci 1996; 63: 425-432.

4. Anal AK, Stevens WF. Chitosan-alginate multilayer beads for controlled release of ampicillin. Int. J. Pharm 2005; 290: 45-54.

5. Shu $X Z$, Zhu KJ. The release behavior of brilliant blue from calcium-alginate gel beads coated by chitosan: the preparation method effect. Eur.J. Pharm. Biopharm. 2002; 53: 193-201.

6. Takka S, Acarturk F. Calcium alginate microparticles for oral administration: I: effect of sodium alginate type and drug release and drug entrapment efficiency. J. Microencapsul 1999; 16: 275-290.

7. Takka S, Omer H, Fu"S, Acartu"RK. Formulation and investigation of nicardipine $\mathrm{HCl}$-alginate gel beads with factorial design-based studies. Eur.J.Pharm 1998; 6: 241-246.

8. Rosario P, Daniela $S$, Maria Angela V, Flavio F, Giovanni $P$. Characterization of the Mechanism of Interaction in Ibuprofen-Eudragit RL 100 Coevaporates. Drug Dev. Ind. Pharm 2004; 30: 277-288. 